\title{
PROGRESS IN CONTACT-BINARY ENERGY TRANSFER
}

\author{
J. A. J. WHE L A N* \\ Institute of Astronomy, Madingley Road, Cambridge CB3 OHA, England
}

Abstract. The energy transfer problem in contact binaries is briefly reviewed.

The need to consider energy transfer between the components of a contact binary comes from the consideration, in the Lucy (1968) model, that the energy radiated by a component is different from the energy generated in its deep interior.

The effects of a source or sink of luminosity in the outer layers of a stellar model have been extensively studied and are moderately well understood; but the exact mechanism of energy transfer remains uncertain. The former aspect of the problem is summarized by Whelan (1972) and references therein; the latter problem has received attention recently from Hazlehurst and Meyer-Hofmeister (1973), Moses (1974), Nariai (1974), Webbink (1975), Yamasaki (1975) and Moses and Smith (1976).

An order of magnitude estimate of the flux of transferred energy, $F$, may be written as $F \sim c_{\mathrm{p}} \rho V \Delta T$ where $c_{\mathrm{p}}$ is the specific heat at constant pressure, $\rho$ the density, $V$ the sideways velocity and $\Delta T$ the temperature excess (Biermann and Thomas, 1972). This estimate shows that deep down in the adiabatic part of the convective envelope where the density is fairly high $\left(\rho \sim 10^{-4} \mathrm{~g} \mathrm{~cm}^{-3}\right)$ sufficient luminosity can be transferred at subsonic velocities. In contrast in the outer superadiabatic layers $\left(\rho \sim 10^{-6.5} \mathrm{~g} \mathrm{~cm}^{-3}\right)$, even at sonic velocities, it is very difficult to carry the necessary flux. Since the exact position of the source or sink of energy is relevant to its effect on stellar parameters a more detailed understanding of the energy exchange mechanism is relevant.

Moses (1974) and Moses and Smith (1976) have considered the details of the sideways energy transfer as a fluid problem with different regimes of length scales and dominant force, while Hazlehurst and Meyer-Hofmeister (1973) have drawn attention to the physical conditions along equipotential surfaces and the size of the entropy gradient between components needed to drive the flow. The sideways flow must consider carefully the heat losses in the neck region connecting the components and the maintenance of a more or less constant temperature over the surface of a component.

Nariai (1974) especially and Hazlehurst and Meyer-Hofmeister (1973) have drawn attention to circulation models which have been considered in detail by Webbink (1975). The possible inefficiencies of the sideways energy transport through the neck have led Webbink (1975) to consider the possibility of a large-scale circulation flow connecting the two components. The flow is driven by the rotation of the stars in analogy with the Eddington-Sweet circulation. The sideways parts of the flow may be inefficient at carrying energy but the vertical parts of the flow may generate or absorb energy due to the $-T \partial S / \partial t$ gravitational energy generation term as matter flows along or against the entropy gradient in the convection zones of the component stars. Order of magnitude estimates indicate that such a vertical flow energy exchange may be capable of generating sufficient luminosity at subsonic flow speeds and a density of $\rho \gtrsim 10^{-7} \mathrm{~g} \mathrm{~cm}^{-3}$.

\footnotetext{
* Present address: Anglo-Australian Observatory, Siding Spring, Australia.
} 
There has been some progress recently in understanding and quantifying the energy exchange process between components of a contact binary system but much work remains to be done.

\section{References}

Biermann, P. and Thomas, H.-C.: 1972, Astron. Astrophys. 16, 60.

Hazlehurst, J. and Meyer-Hofmeister, E.: 1973, Astron. Astrophys. 24, 379.

Lucy, L. B.: 1968, Astrophys. J. 151, 1123.

Moses, A. P.: 1974, D.Ph. Thesis, University of Sussex.

Moses, A. P. and Smith, R. C.: 1976 (this volume, p. 333).

Nariai, K.: 1974, paper presented at the Liège Conference on Dynamical Processes in Stars.

Webbink, R. F.: 1975, Ph.D. Thesis, University of Cambridge.

Whelan, J. A. J.: 1972, Monthly Notices Roy. Astron. Soc. 156, 115.

Yamasaki, A.: 1975, Publ. Astron. Soc. Japan (in press).

\section{DISCUSSION}

Shu: The problem you describe is exceptionally difficult - comparable to the problem of planetary scale circulation patterns in the Earth's atmosphere. The problem is that you have a situation where heat comes out from the bottom at different rates; the analogue in the Earth is the different rates of heating at equator and pole. The redistribution of heat horizontally while maintaining a minimum amount of horizontal pressure difference is very complex, as you well know.

Whelan: I agree.

Shaviv: I would like to amplify Dr Shu's comment. Since opacity increases inward in these outer layers, heat will tend to flow along the meridian rather than inward. These heat currents will form large meridional circulation.

Whelan: The Webbink model appeals to large-scale circulation. 